\title{
AutoBayes: A System for Generating Data Analysis Programs from Statistical Models
}

\author{
BERND FISCHER and JOHANN SCHUMANN \\ RIACS / NASA Ames, Moffett Field, CA USA \\ (e-mail: $\{$ fisch, schumann $\}$ @ptolemy.arc.nasa.gov)
}

\begin{abstract}
Data analysis is an important scientific task which is required whenever information needs to be extracted from raw data. Statistical approaches to data analysis, which use methods from probability theory and numerical analysis, are well-founded but difficult to implement: the development of a statistical data analysis program for any given application is time-consuming and requires substantial knowledge and experience in several areas.

In this paper, we describe AutoBAyEs, a program synthesis system for the generation of data analysis programs from statistical models. A statistical model specifies the properties for each problem variable (i.e., observation or parameter) and its dependencies in the form of a probability distribution. It is a fully declarative problem description, similar in spirit to a set of differential equations. From such a model, AutoBayes generates optimized and fully commented $\mathrm{C} / \mathrm{C}++$ code which can be linked dynamically into the Matlab and Octave environments. Code is produced by a schema-guided deductive synthesis process. A schema consists of a code template and applicability constraints which are checked against the model during synthesis using theorem proving technology. AutoBAYEs augments schema-guided synthesis by symbolic-algebraic computation and can thus derive closed-form solutions for many problems. It is well-suited for tasks like estimating best-fitting model parameters for the given data. Here, we describe AuTOBAYES's system architecture, in particular the schema-guided synthesis kernel. Its capabilities are illustrated by a number of advanced textbook examples and benchmarks.
\end{abstract}

\section{Introduction}

Data analysis denotes the transformation of raw data (i.e., pure numbers) into a more abstract form, e.g., summarizing a set of measurements by their mean value and standard deviation. For most data analysis tasks - especially tasks involving large data sets - computer support is necessary. Consequently, scientists of all disciplines spend much time writing and changing data analysis programs, ranging from simple, straight-forward (e.g., linear regression) to truly complex (e.g., image analysis systems to detect new planets). However, the manual development of a customized data analysis program for any given application problem is not only time-consuming but also error-prone. It requires a rare combination of profound expertise in several areas - computational statistics, numerical analysis, software engineering, and of course the application domain itself. We believe that the application of program generation techniques can help to counter these difficulties. 
In this paper, we describe AutoBAyes, a program generator for scientific data analysis programs.

Scientific data analysis is usually based on statistical methods. The expected properties of the data are described in the form of a statistical model: for each problem variable (i.e., observation or parameter), properties and dependencies are specified via probability distributions. In many applications, an initial statistical model of the data is readily available, but the parameters of the model (e.g., mean values and variances) are unknown. Then, a typical data analysis task is to fit observed data against the model, i.e., to find the best possible or most likely values of the unknown parameters under the constraints specified by the model. Here we concentrate on generating programs for such parameter learning tasks.

AutoBayes starts from a very high-level description of the data analysis problem in the form of such a statistical model and generates imperative programs through a schema-based deductive synthesis process. A schema is a code template with associated semantic constraints which define and restrict the template's applicability. The schemas are applied recursively to the entire problem or subproblems. AutoBAyes augments this schema-based approach by symbolic-algebraic calculation and simplification to derive closed-form solutions for the entire problem (or subproblems) whenever possible. This is a major advantage over other statistical data analysis systems which have to use slower and possibly less precise numerical approximations even in cases where closed-form solutions exist. The backend of AutoBAyes is designed to support generation of code for different programming languages and different target systems. Our current version generates $\mathrm{C} / \mathrm{C}++$ code which can be linked dynamically into the Octave or Matlab environments; other target systems can be added easily.

We believe that data analysis is a generally very promising application area for program generation. On the one hand, the domain itself is well-suited. Probability theory provides an established, domain-specific notation for the statistical models which can form the basis of a specification language. Statistical models are fully declarative problem descriptions in this notation; they specify properties and dependencies of the problem variables but do not prescribe any specific algorithms. Moreover, probability theory and numerical analysis provide a wide variety of solution methods and potentially applicable algorithms. On the other hand, the potential pay-off of program generation is huge. Manual development of data analysis programs is a skill-intensive, time-consuming and error-prone task. Algorithm libraries are only of limited help as the algorithms need to be customized, optimized, and appropriately packaged before they can be integrated. Most importantly, the development process for data analysis programs is typically highly iterative: the underlying model is usually changed many times before it is suitable for the application; often the need for these changes becomes apparent only after an initial solution has been implemented and tested on application data. However, since even small changes in the model can lead to entirely different solutions, e.g., requiring a different approximation algorithm, developers are often reluctant to change (and thus improve) the model and settle for sub-optimal solutions. For example, the data analysis routines of the TOMS ozone spectrometer were over-simplified to ignore 


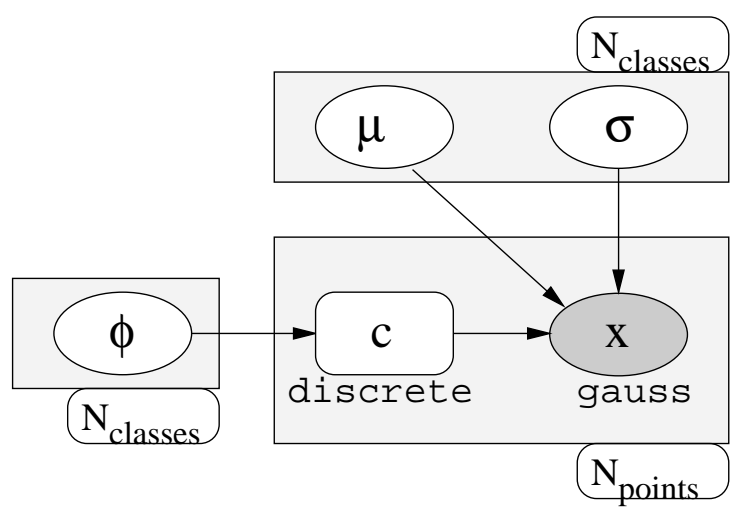

Fig. 1. Bayesian network for the mixture of Gaussians example

very low ozone readings, thus delaying the detection of the ozone hole over the Antarctic by several years (Centre for Atmospheric Science, 1999). Automated program synthesis can help to solve such problems. It encapsulates a considerable part of the required expertise and allows the developers to program in models, thereby increasing their productivity. By automatically generating code from these models, many programming errors are prevented and turn-around times are reduced.

This paper is an extended version of (Fischer et al., 2000); design rationales and some preliminary results of the AUTOBAYES-project have also been reported in (Buntine et al., 1999). Section 2 contains a very short introduction into probabilistic and graphical reasoning; we refer to the cited literature for more information. Section 3 explains the mixture of Gaussians model in more detail; that model is used as the running example throughout this paper. We then proceed in Sections 4 and 5 with a detailed description of the system architecture and the code synthesis process. Section 6 illustrates in detail the derivation of code for the running example; it also contains an overview of a number of other example problems solved by AutoBayes. We compare our approach to related work in Section 7 before we concluded and discuss future work in Section 8.

\section{Probabilistic and Graphical Reasoning}

Graphical models such as Bayesian networks are a common representation method in machine learning and statistical data analysis (Pearl, 1988; Buntine, 1994; Frey, 1998; Jordan, 1999). They combine probability theory and graph theory. From a computational point of view, their appeal is that they can replace some expensive probabilistic reasoning by faster graphical reasoning.

AutoBayes uses a version of hybrid Bayesian networks to represent the specified statistical model internally; Figure 1 shows a network for the mixture of Gaussians example which we use throughout this paper. A Bayesian network is a directed, acyclic graph whose nodes represent random variables and whose edges define probabilistic dependencies between the random variables. In a hybrid Bayesian network 
nodes can represent discrete as well as continuous random variables; these are usually represented by boxes and circles, respectively. In the example, $c$ is the single discrete random variable while $\mu, \sigma, \phi$, and $x$ are all continuous random variables. Shaded nodes represent known variables, i.e., input data; here, only $x$ is known. Distribution information for the random variables is attached to the respective nodes; here, $x$ is distributed as a Gaussian. Lightly shaded boxes enclosing a set of nodes represent vectors of independent, co-indexed random variables. In the example, $\mu$ and $\sigma$ are both vectors of size $N_{\text {classes }}$ which always occur indexed in the same way. As a consequence, a box around a single node represents the familiar concept of a vector of independent and identically distributed variables.

The edges in a Bayesian network can sometimes be interpreted as causal influence links between the respective variables. For example, the edge from $\mu$ to $x$ represents the influence the (hypothetical) choice of $\mu$ has on the observed data $x$. More precisely, however, the edges encode a conditional independence relationship: each node is independent of its ancestors given its parents. In the example, $x$ is thus independent of $\phi$ given $c, \mu$, and $\sigma$. Consequently, the conditional probability $P(x \mid$ $c, \phi, \mu, \sigma)$ is equal to - and can thus be simplified to- $P(x \mid c, \mu, \sigma)$. The network thus superimposes a structure on the global joint probability distribution which can be exploited to optimize probabilistic reasoning. Hence, the example defines the joint probability $P(x, c, \phi, \sigma, \mu)$ in terms of simpler probabilities:

$$
P(x, c, \phi, \sigma, \mu)=P(\phi) \cdot P(c \mid \phi) \cdot P(\mu) \cdot P(\sigma) \cdot P(x \mid c, \mu, \sigma)
$$

Probabilistic reasoning is currently subject to a - sometimes heated - debate between two different schools of thought, the so-called "frequentist" and "Bayesian" approaches. The basic difference between the two approaches is their view of probability. In the frequentist approach, a probability is viewed as a relative frequency which is the outcome of a long series of repeated identical experiments. In a strictly frequentist sense no inference can thus be made based on single events. In the Bayesian approach, a probability is viewed as a degree of belief that an event occurs. Prior beliefs and knowledge of the state of the analyzed system are specified by prior distributions or priors for short. New data is then considered evidence which is combined with the priors, using Bayes rule

$$
P(h \mid d)=\frac{P(d \mid h) \cdot P(h)}{P(d)}
$$

The posterior probability $P(h \mid d)$ that the hypothesis $h$ holds under the new data $d$ is thus expressed in terms of the likelihood $P(d \mid h)$ and the prior $P(h)$; the probability of the data, $P(d)$, is a normalizing constant. Despite these fundamental differences in interpretation, the techniques applied in both approaches are quite similar. A frequentist analysis can usually be simulated in the Bayesian approach by choosing an appropriate non-informative prior, or, intuitively, by leaving the model parameters uninterpreted. AutoBAYEs can thus be used as a tool in both approaches; the preference for a particular approach is reflected in the formulation of the statistical model only.

Graphical methods can be applied to two different kinds of data analysis prob- 
(a)

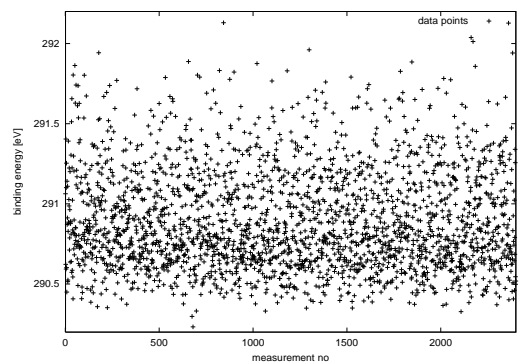

(b)

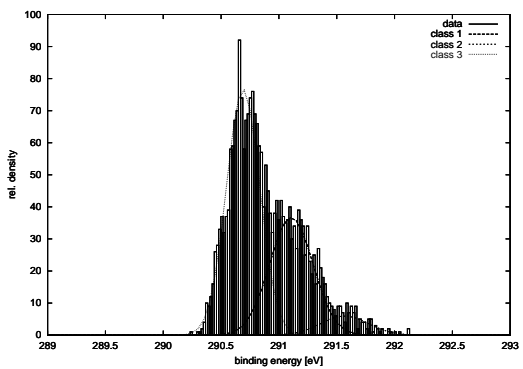

Fig. 2. (a) Artificial input data for the mixture of Gaussians example: 2400 data points in the range $[290.2,292.2]$. Each point belongs in one of three classes which are Gaussian distributed with $\mu_{1}=290.7, \sigma_{1}=0.15, \mu_{2}=291.13, \sigma_{2}=0.18$, and $\mu_{3}=291.55, \sigma_{3}=$ 0.21 . The relative frequencies $\phi$ for the points belonging to the classes are $61 \%, 33 \%$, and $6 \%$, respectively. (b) Histogram (spectrum) of the artificial test data from (a) and Gaussian distributions which are obtained as the result of the synthesized data analysis program.

lems. In the first case, parameter learning, both data and model are given and the parameters of the model (in our example $\mu, \sigma$, and $\phi$ ) have to be determined. In the second case, structure learning, only the data is given, and both the model and its parameters have to be determined. This involves a usually heuristic search in the space of all models, e.g., using a hill climbing method. Within this search, parameter learning usually re-appears as a subtask. Currently, AutoBAYES is set up to handle parameter learning only - it requires the (parameterized) model specification as input. However, it can in principle also be employed in the inner loop of a structure learning algorithm.

Parameter learning is basically an optimization problem. In some cases, closed form solutions for the optimal parameter values exist and the equations derived from the network structure and the probability density functions can be solved symbolically. In general, however, iterative methods must be applied to solve the optimization problem. Typically, learning and classification algorithms as for example $k$-Means or expectation maximization (EM) are used. For some subproblems, classical numerical optimization algorithms like Newton, Gauss-Newton, or other variants are applicable.

\section{An Example: Mixture of Gaussians.}

Throughout this paper, we will illustrate how AuTOBAYEs works by means of a simple but realistic classification example. Figure 2(a) shows the raw input data, a vector of real values. We know that each data point falls into one of three classes; each class $i$ is Gaussian distributed with mean $\mu_{i}$ and standard deviation $\sigma_{i}$. The data analysis problem is to infer from the given data the relative class frequencies $\phi_{i}$ (i.e., how many points belong to each class) and the unknown distribution parameters $\mu_{i}$ and $\sigma_{i}$ for each class.

This example and its underlying model are deliberately rather simple but the model can already be used in several applications. Berkowitz (Berkowitz, 1979) 


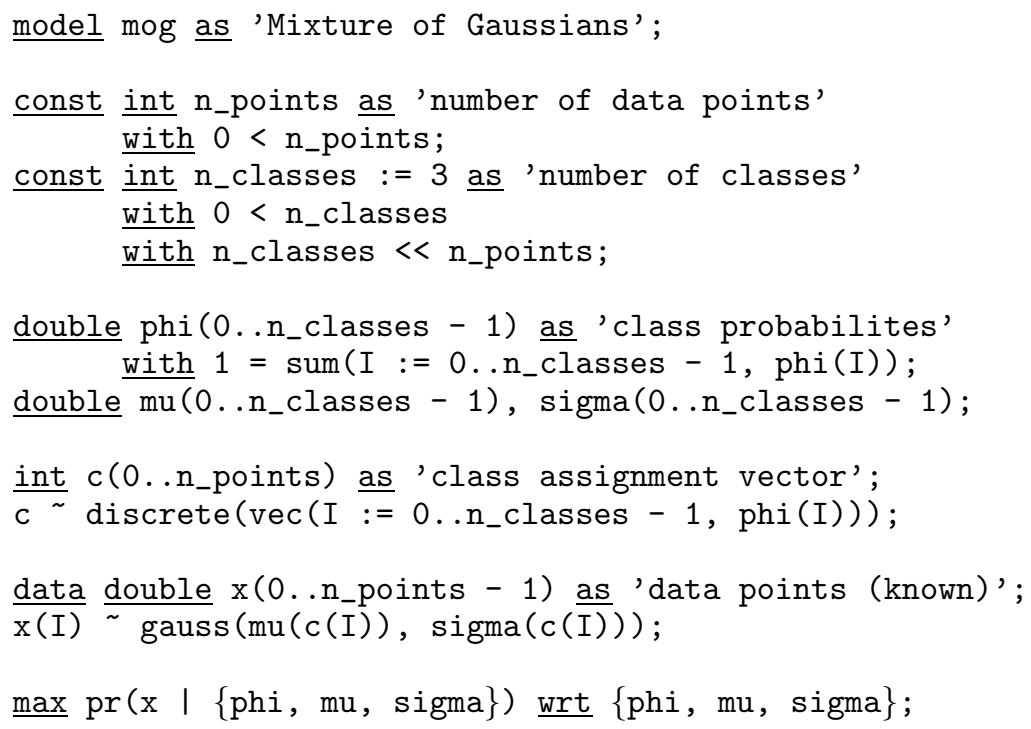

Fig. 3. AutoBAyes-specification for the mixture of Gaussians example. Line numbers have been added for reference in the text. Keywords are underlined.

describes an application in physics where gas atoms (or molecules) are excited with a specific energy (e.g., light from a laser). They can then absorb this energy by excitation or electron emission. This basic mechanism generates spectral lines like those observed in the light of stars. Single atoms usually have sharp, welldefined spectral lines but the more complex molecules (e.g., $\mathrm{CH}_{4}$ or $\mathrm{NH}_{3}$ ) can have several peaks of binding energy, depending on their internal configuration. Thus, they can absorb (or emit) energy at different levels. Figure 2(b), which is adapted from (Berkowitz, 1979), shows an spectrum of the energy of emitted photoelectrons which is directly related to the excess energy of photons over the photoionization potential of $\mathrm{CH}_{4}$ molecules (for details see (Berkowitz, 1979), caption of Figure 67). Since $\mathrm{CH}_{4}$ which has three internal distinct configurations, the spectrum shows three distinct peaks.

In a simple statistical model, each of the peaks is assumed to be independently Gaussian distributed and the percentage of molecules in a specific configuration is assumed to be known. When we measure the binding energies for a large number of $\mathrm{CH}_{4}$ molecules (with unknown internal configurations), we obtain a data set similar to the one shown in Figure 2(a). We can then use a program implementing the statistical model to classify the data points into the three classes and to obtain the parameters. Figure 2(b) shows the histogram of the data, superimposed with Gaussian curves using the parameter values estimated by the program generated by AutoBayes.

Figure 3 shows the detailed statistical model for this problem in AutoBAYES's specification language. The model (called "Mixture of Gaussians" - line 1) assumes that each of the data points (there are n_points - line 5) belongs to one of $\mathrm{n}_{-}$classes classes; here $\mathrm{n}_{-}$classes has been set to three (line 3 ), but $\mathrm{n}_{-}$points is 
left unspecified. Lines 16 and 17 declare the input vector and distributions for the data points. ${ }^{1}$ Each point $\mathrm{x}(\mathrm{I})$ is drawn from a Gaussian distribution $\mathrm{c}$ (I) with mean $\mathrm{mu}(\mathrm{c}(\mathrm{I}))$ and standard deviation sigma(c(I)). The unknown distribution parameters can be different for each class; hence, we declare these values as vectors (line 11). The unknown assignment of the points to the classes (i.e., distributions) is represented by the hidden (i.e., not observable) variable c corresponding to the internal configuration of the molecule. The class probabilities or relative frequencies are given by the also unknown vector phi (lines 9-14). Since each point belongs to exactly one class, the sum of the probabilities must be equal to one (line 10). Additional constraints (lines 4, 6,7) express further basic assumptions of the model. Finally, we specify the goal inference task (line 19), maximizing the conditional probability $\operatorname{pr}(\mathrm{x} \mid\{\mathrm{phi}, \mathrm{mu}$, sigma $\})$ with respect to the parameters of interest, phi, mu, and sigma. This means, we are interested in obtaining the values for the model parameters which best fit the given data.

This classification problem is a typical task in (unsupervised) machine learning for which a variety of algorithms and approaches exist (see, e.g., (Mitchell, 1997; Bishop, 1995)). AutoBAyEs currently implements two such algorithms which are known in the literature as $k$-Means and expectation maximization or simply EM algorithm (Dempster et al., 1977; McLachlan \& Krishnan, 1997), respectively. Both algorithms are applicable to a variety of mixture models (McLachlan \& Peel, 2000) which underpin many classification tasks similar to our running example.

The EM-algorithm is an iterative numerical algorithm which applies to maximization tasks of the form $\max P(U \mid V)$ wrt $V$, given a set $W$ of hidden variables. In our example, $U=\{\mathrm{x}\}, V=\{\mathrm{phi}, \mathrm{mu}$, sigma $\}$, and $W=\{\mathrm{c}\}$. The algorithm basically consists of three steps; the first step performs initializations. In our implementation, the initialization just "guesses" values for the hidden variables by performing random assignments. These assignments are made to a matrix $q$ where $q(i, j)$ is the probability that point $i$ belongs to class $j$. Then an iteration is performed over the remaining two steps, the expectation or E-step, and the maximization or $M$ step. This iteration is performed until the changes of the involved variables become sufficiently small. During the iteration, $E$-step and $M$-step change the position of the distribution parameters.

- M-step: given the current distribution of $W$ (in our example, the values of the matrix q and the data $U)$, new values for the distribution parameters $V$ are estimated by maximizing $P(\{W, U\} \mid V)$ with respect to $V$. In our example, this maximization results in new estimates of mu and sigma for each of the classes.

- E-step: given the current estimated values for the distribution parameters $V$ and the data $U$, the probability distribution of $W$ is calculated. In the discrete case, as in our example, this distribution can be obtained relatively easily by summing up over the domain of $W$. Thus, in our case, we update the matrix $\mathrm{q}$ to reflect the new estimates of the parameters.

\footnotetext{
${ }^{1}$ Vector indices start with 0 in a $\mathrm{C} / \mathrm{C}++$ style.
} 
The individual steps of this generic algorithm need to be adapted for the specific model. For example, the maximization step requires information about the distribution of all variables and involves substantial symbolic calculations (e.g., as shown in the for-loop near the bottom of Figure 7).

\section{System Architecture}

\subsection{Overview}

AutoBAYES's overall system architecture is shown in Figure 4. In a first processing step, the given specification is parsed and converted into internal form and the Bayesian network is constructed. This step can also generate an external representation for visualization purposes, using the dot graph drawing tool (Koutsofios \& North, 1996). The synthesis kernel, which will be described in detail in Section 5, then analyzes the network, tries to solve the given optimization task, and instantiates appropriate algorithm schemas which are given in a schema library. The output of the synthesis kernel is a program in a procedural intermediate language. AutoBAYES's backend (see Section 4.2) takes this intermediate code, optimizes it and generates code for the chosen target system. Currently, we target Octave and Matlab but only small parts of the code generator are system-specific; new target systems can thus be added easily. The synthesis kernel also produces detailed documentation along with the code (see Section 4.3). Furthermore, AutoBayes can generate code which generates artificial data for the model, e.g., for visualization and testing purposes (see Section 4.4).

All parts of the AutoBAYes system rely heavily on a symbolic subsystem and some auxiliary system modules (e.g., pretty-printer, set representations, I/O functions). For symbolic mathematical calculations, we implemented a small but reasonably efficient rewriting engine in Prolog. Graph handling, simplification of mathematical expressions, and an equation solver are implemented on top of it. The system architecture is designed in such a way that most of its parts can be re-used in different domains. In particular, backend and symbolic subsystem are entirely independent of the data analysis domain. The entire system has been implemented in SWI-Prolog (Wielemaker, 1998) and comprises about 31,000 lines of documented Prolog code. Since AutoBAyEs requires a combination of sound symbolic mathematical calculation, rewriting, and general purpose operations (e.g., output to multiple files, handling of strings, interface to the operating system), Prolog is a reasonable choice as the underlying implementation language. SWI-Prolog proved

to be a very stable and efficient development platform with reasonable debugging facilities.

\subsection{Generating Code}

The synthesis kernel of AutoBAYES generates code in an intermediate language before the code for the actual target system is produced. This intermediate language is a simple procedural language with several domain-specific extensions as for example convergence loops, vector normalization, simultaneous vector assignment, and 


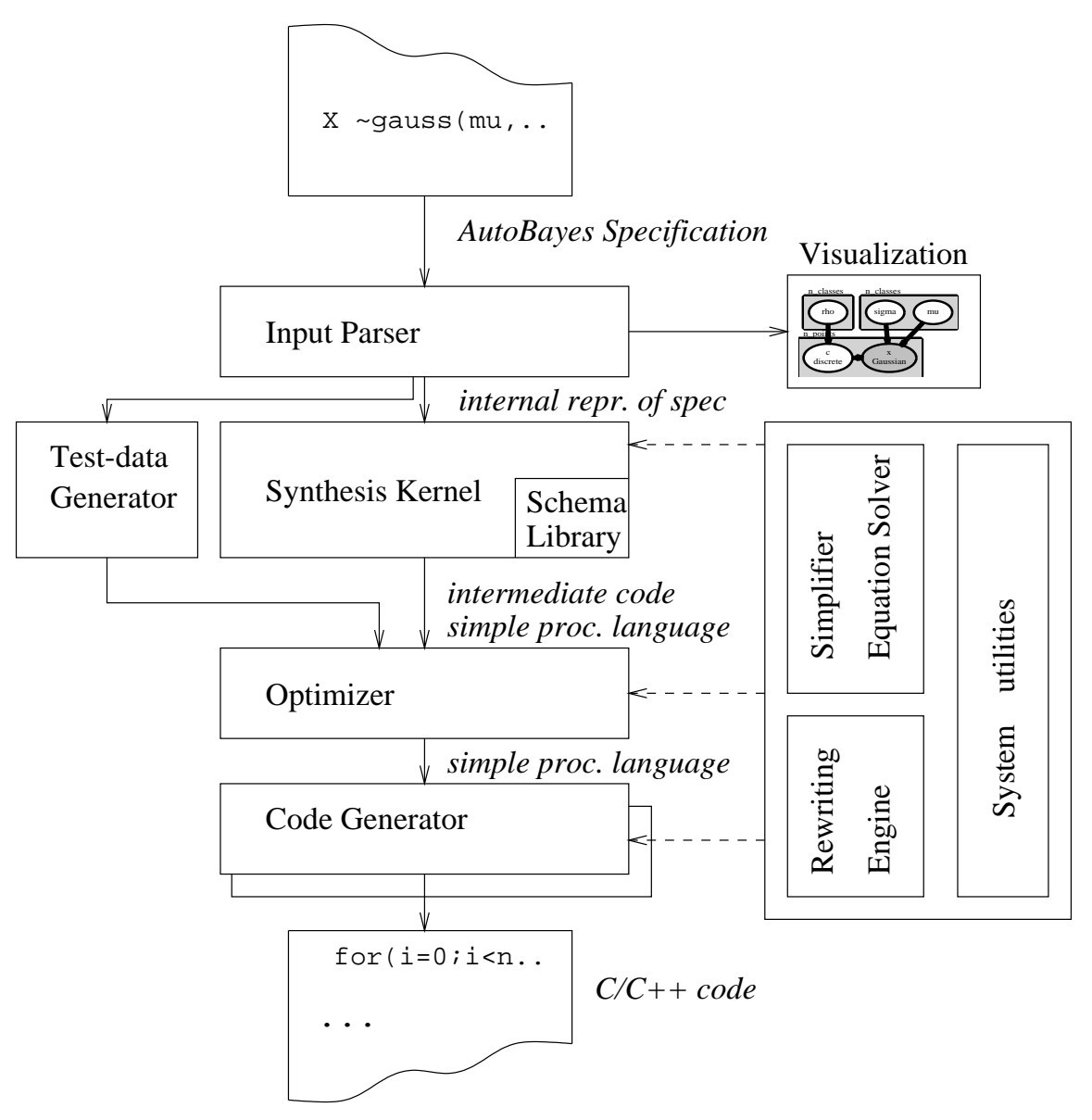

Fig. 4. System architecture of AutoBayes.

assertions and annotations. The intermediate language is still close enough to the current target languages (i.e., $\mathrm{C}$ and $\mathrm{C}++$ ) such that the translation down into the chosen target language remains simple. The domain-specific constructs allow target-specific optimizations and transformations. For example, the sum-construct of the intermediate language for calculating the sum of array elements can be converted into a usual for-loop, an iterator construct for sparse matrices, or a direct call to a summation-operator (e.g., when generating interpreted Matlab code).

The actual target-specific portion of the code-generator is rather straight-forward and can be adapted to different target languages and environments. With the help of rewrite rules all constructs of the intermediate language are transformed into constructs of the target language and printed using a generic pretty-printer. The backend also generates boilerplate code to interface the algorithm with the target system, and to check for correct types of arguments. The synthesized code is also optimized. However, standard optimizations (e.g., evaluation of constant expres- 
sions) are left for the subsequent compilation phase - there is no need to perform the same optimization steps as any modern compiler.

The current AutoBAYES-version generates C++-code for Octave (Murphy, 1997), C-code for Matlab (Moler et al., 1987), and stand-alone C-code. Future work will include code-generators for CASE-tools for embedded systems, e.g., ControlShell (ControlShell, 1999) or MatrixX (AutoCode, 1999).

\subsection{Generating Documentation}

Certification procedures for safety-critical applications (e.g., in aircraft or spacecraft) often mandate manual code inspection. This inspection requires that the code is readable and well documented. Even for programs not subject to certification, understandability is a strong requirement as manual modifications are often necessary, e.g., for performance tuning or system integration. However, existing program generators often produce code that is hard to read and understand. In order to overcome this problem, AUTOBAYES generates explanations along with the programs which show the "synthesis decisions": which algorithm schema has been used, how the schema parameters have been instantiated, etc. Used model assumptions and proof obligations that could not be discharged during the synthesis are laid out clearly. This makes the synthesis process more transparent and provide traceability from the generated program back to the model specification.

AutoBAYEs generates extensively commented code: approximately one third of the output lines are automatically generated comments (cf. Figure 7 for an example). This is achieved by embedding documentation templates into the code templates. Future versions of AUTOBAYES will not only generate fully documented code; we aim to produce a detailed standardized design-document for the generated code.

\subsection{Generating Artificial Test Data}

Visualization and simulation plays an important role in the development of data analysis programs. An AUtoBAYES model specification contains enough information to synthesize code which generates artificial data according to the specification. For example, the data set in Figure 2(a) has been generated that way. Generating artificial test data is very helpful in understanding the model and the generated code. If the artificial data does not match real data sets (or the scientist's expectations), the specified model might not reflect the reality properly. Artificial data sets can also be used to assess and evaluate the performance of the synthesized code before real data becomes available. This feature is of particular interest in cases where the domain theory allows instantiation of different algorithms for the same specification. For example, if AutoBAYES synthesizes different variants for initialization of the hidden variable, their coarse relative performance can be assessed with the generated test data.

For AutoBayes, we have developed a module which can synthesize a program to generate artificial data according to the given specification. This specification 
is exactly the same as for the synthesis of the data analysis program. This data generator was implemented in less than 200 lines of Prolog code on top of the AutoBayes system.

\section{The Synthesis Kernel}

\subsection{Network Construction}

The synthesis kernel takes the internal representation of the model specification and builds an initial Bayesian network. Each variable declaration in the model corresponds directly to a network node. Each distribution declaration of the form $x \sim D(\Theta)$ (for any distribution $D$ ) induces edges from the distribution's parameters $\Theta$ to the node corresponding to the random variable $x$; these edges reflect the dependency of the (random) values of $x$ on the values of the parameters $\Theta$. Building the network is relatively straight-forward and requires no sophisticated dataflow analysis because the model is purely declarative. However, $\Theta$ needs to be flattened, i.e., nested random variables need to be lifted and fresh index variables need to be introduced in their place in order to represent the dependencies properly. Hence, the example declaration $x(I) \sim \operatorname{gauss}(\mathrm{mu}(\mathrm{c}(\mathrm{I}))$, sigma $(\mathrm{c}(\mathrm{I})))$ induces not only the two obvious edges but three: $\mathrm{mu}(\mathrm{J}) \longrightarrow \mathrm{x}(\mathrm{I})$, sigma $(\mathrm{J}) \longrightarrow \mathrm{x}(\mathrm{I})$, and $\mathrm{c}(\mathrm{I})$ $\longrightarrow \mathrm{x}(\mathrm{I})$ (cf. Figure 1). Note that $\mathrm{x}$ and $\mathrm{c}$ are still co-indexed but that each $\mathrm{x}(\mathrm{I})$ now depends on all $\mathrm{mu}(\mathrm{J})$ and sigma $(\mathrm{J})$, reflecting the unknown values of their original indices $\mathrm{c}(\mathrm{I})$. A compact representation of the indexed nodes and their dependencies is achieved by using Prolog-variables to represent index variables.

\subsection{Schema-Guided Synthesis}

Synthesis proceeds from this initial network and the original probabilistic inference task by exhaustive application of schemas. A schema can be understood as an "intelligent macro": it comprises a pattern, a parameterized code template, and a set of preconditions or applicability constraints. The pattern and code template are similar to the left- and right-hand side of a traditional macro definition; they comprise the syntactic part of the schema. Schema-guided synthesis, however, is not just macro expansion. Different schemas can match the same pattern, possibly in different ways. During synthesis, these schemas are tried exhaustively in a left-to-right, depth-first manner. Whenever a dead end is encountered (i.e., no schema is applicable), AutoBayes backtracks. This control regime allows AutoBayes to generate code as a composition of different schemas, thus "re-inventing" data-analysis algorithms from simple building-blocks. Furthermore, backtracking in AutoBayes results in the synthesis of program variants if multiple schemas are applicable and thus yields the capability to generate multiple solutions for the same problem.

The constraints of a schema refine its semantics: a schema can be understood as an axiom which asserts that the program (i.e., the appropriately instantiated template) solves the probabilistic inference task specified by the pattern if the constraints are satisfied; however, checking the constraints may instantiate the template 
parameters further. The search process mentioned above is thus a proof search; the proof is constructive in the sense that it actually generates a program (the witness) and does not just assert its existence.

Network decomposition schemas. AutoBAyes uses four different kinds of schemas. Network decomposition schemas are encodings of independence theorems for Bayesian networks (see for example (Pearl, 1988)). They describe how a probabilistic inference task over a given network can be decomposed equivalently into simpler tasks over simpler networks and, hence, how a complex data analysis program can be composed from simpler components. The applicability constraints for these schemas can be checked by pure graph reasoning. Consider for example the following decomposition theorem:

Let $U, V$ be sets of vertices in a Bayesian network such that $U \cap V=\emptyset$. Then $V \cap \operatorname{descendants}(U)=\emptyset$ and parents $(U) \subseteq V$ implies

$$
\begin{aligned}
P(U \mid V) & =P(U \mid \operatorname{parents}(U)) \\
& =\prod_{u \in U} P(u \mid \text { parents }(u))
\end{aligned}
$$

This theorem allows us to simplify the conditional probability $P(U \mid V)$ into $P(U \mid$ parents $(U))$. This means that we can safely ignore all assumptions not reflected in the network by incoming edges. Then $P(U \mid$ parents $(U))$ can further be decomposed into a finite product of atomic probabilities (i.e., each variable depends only on the parameters of its associated distribution), provided that the applicability constraints hold over the network; here, descendants $(U)$ is the set of all nodes (directly or indirectly) reachable from nodes in $U$ excluding $U$. Within AutoBAYEs, this theorem is implemented by the following network decomposition schema for maximizing the probability $P(U \mid V)$ with respect to a set of variables $X$ :

$$
\begin{aligned}
\text { schema }( & \underline{\max } P(U \mid V) \underline{\text { wrt }} X, \text { Template }):- \\
& U \cap V=\emptyset \\
& \wedge V \cap \text { descendants }(U)=\emptyset \\
& \wedge \text { parents }(U) \subseteq V \wedge \ldots \\
& \rightarrow \text { Template }=\underline{\text { begin }} \\
& \underline{\text { end }}
\end{aligned}
$$

The schemas are written as Prolog-rules. During the search for applicable schemas, pattern-matching with the rule head (first line) is attempted. When the match succeeds, the schema variables $(U, V$, and $X)$ are bound, and the body of the rule (separated by the :- from the head) is processed. Here, the body is a logical implication. The implication's antecedents directly encode the applicability constraints as AUTOBAYES's symbolic reasoning engine contains an operationalization of the graph predicates. The schema's code template consists of a code fragment bracketed by begin and end. Its body is a sequence of simpler maximization tasks which are solved by recursive calls to the synthesizer. Their ordering is irrelevant because the $u \in U$ are independent of each other; this is a consequence of the applicability constraints. 
In our ongoing example, this decomposition schema is applied when the intermediate goal $\underline{\max } \operatorname{pr}(\{\mathrm{c}, \mathrm{x}\} \mid\{\mathrm{phi}, \mathrm{mu}, \mathrm{sigma}\})$ wrt $\{\mathrm{phi}, \mathrm{mu}$, sigma $\}$ is processed. With $U=\{\mathrm{c}, \mathrm{x}\}, V=\{\mathrm{phi}, \mathrm{mu}, \mathrm{sigma}\}$, and $X=\{\mathrm{phi}, \mathrm{mu}, \mathrm{sigma}\}$, it is easy to see that all requirements for the schema are satisfied (see Figure 1 for the dependencies among the variables). Thus, we obtain the following two (simpler) maximization goals: $\max \operatorname{pr}(\mathrm{c} \mid \mathrm{phi})$ wrt $\{\mathrm{phi}$, and $\underline{\max } \operatorname{pr}(\mathrm{x} \mid\{\mathrm{c}, \mathrm{mu}$, sigma $\}$ ) wrt $\{\mathrm{mu}$, sigma $\}$.

A number of similar decomposition theorems have been developed in probability theory; AutoBAYES currently includes three different schemas based on such theorems, with the one shown above being by far the simplest. For details on the other schemas see (Buntine et al., 1999).

Formula decomposition schemas. Formula decomposition schemas are similar to the network decomposition schemas above but they work on complex formulae instead of a single probability. The following schemas are typical members of this class.

- Index decomposition applies to an inference task for a formula which contains multiple occurrences of probabilities involving vectors and "unrolls" this task into a loop over the simpler inference task for a single vector element. In our example, one subtask is max $\operatorname{pr}(\mathrm{x} \mid\{c, \mathrm{mu}$, sigma $\})$ wrt $\{\mathrm{mu}$, sigma $\}$. Since the vector $\mathrm{x}$ is independently and identically distributed (i.e., has the same distribution for each data point), maximization can be done separately for each I. Thus, we obtain the code fragment for $i=0$..n_points-1 : $\langle\underline{\max }$ $\operatorname{pr}(x(I) \mid\{c(I), m u$, sigma $\})$ wrt $\{p h i(i)\}\rangle$.

- Split/back-substitute splits a mixed discrete-continuous maximization problem into two separate discrete and continuous subproblems, respectively, and substitutes a symbolic solution of the continuous subproblem back into the discrete subproblem.

- Iterate-range: solves a discrete maximization problem by iteration over the finite range of the variables.

Most of the applicability constraints for these decomposition schemas can still be checked by graph reasoning but some checks involving the formula structure require substantial symbolic reasoning.

Statistical algorithm schemas. Proper statistical algorithm schemas are also graph-based but they are not simple consequences of the independence theorems. These schemas involve larger modifications of the graph, e.g., introduction of new nodes with known values, and storing the results of intermediate calculations. These schemas thus enable the further application of the decomposition schemas; however, they are much more intricate and less theorem-like. Hence, their correctness is proven independently, or they are just empirically validated during construction of the domain theory. Statistical algorithm schemas also have much larger and usually iterative code templates associated with them and they can require substantial symbolic reasoning during instantiation. AUTOBAYEs currently implements two such algorithms, namely $k$-Means and the EM algorithm.

As already described in Section 3, the EM-algorithm schema applies to maxi- 
mization tasks of the form $\underline{\max } P(U \mid V)$ wrt $V$, given a set $W$ of hidden variables. Within AutoBAyEs, EM is encoded as the following schema:

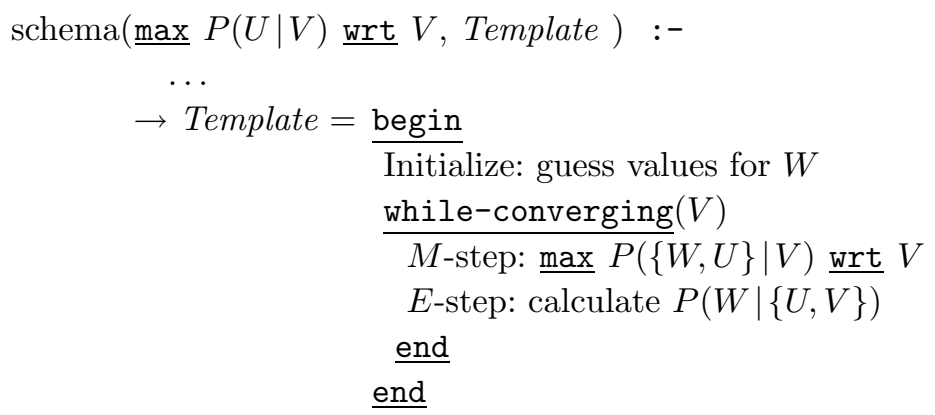

Each of the three steps (initialization, $M$-, and $E$-step) cause recursive calls to the synthesizer. The maximization task in the $M$-step triggers further decompositions by the assumption that the hidden variables $W$ are now known.

Numerical algorithm schemas. The graph-based reasoning continues until all conditional probabilities $P(U \mid V)$ have been converted into atomic form, i.e., parents $(U)=V$. This means that all random variables occurring in the parameters of $U$ 's (joint) distribution are known. Such probabilities can thus be replaced by the appropriately instantiated probability density functions. AuTOBAYEs's domain theory contains rewrite rules for the most common probability density functions. In our example, $\operatorname{pr}(x(i) \mid\{m u(j), \operatorname{sigma}(j)\}$ is rewritten into

$$
(\sqrt{2 \pi} \operatorname{sigma}(j))^{-1} \exp \left(\frac{(\mathrm{x}(\mathrm{i})-\operatorname{mu}(j))^{2}}{-2 \operatorname{sigma}(j)^{2}}\right)
$$

thus instantiating the usual formula for Gaussian distributions. Density functions for problem-specific distributions can easily be defined as part of an AUTOBAYES specification.

With this elimination step the original probabilistic inference task becomes a pure optimization problem which can be solved either symbolically or numerically. AutoBayes first attempts to find closed-form symbolic solutions, which are much more time-efficient during run-time than iterative numeric approximation algorithms. In order to solve the optimization problem, AUTOBAYES symbolically differentiates the formula with respect to the optimization variables, sets the result to zero and tries to symbolically solve this system of simultaneous equations. Symbolic differentiation is implemented as a term rewrite system; however, some variable dependency checks require conditional rewrite rules. For example, it has to be checked whether the dependent variable of the derivative occurs in a term or not. Equation solving currently employs only a variant of Gaussian variable elimination; whenever a variable can be isolated modulo the symbolic model constants, the remaining equation is solved by a polynomial solver.

If no symbolic solution can be found, AutoBAyes applies iterative numerical optimization algorithm schemas, e.g., as described in (Press et al., 1992; Gill et al., 1981) and found in most general-purpose numeric libraries. The current version of AutoBayes incorporates the Newton-Raphson and the Nelder-Mead simplex methods. However, program synthesis can substantially improve the black-box style 
reuse typical for libraries. It can instantiate actual parameters symbolically and evaluate the inlined expressions partially. This provides further optimization opportunities, often in the inner loops of the algorithms. Moreover, symbolic and numeric methods complement each other well. While for many more complex models no complete closed-form solutions exist, AutoBAYes can usually solve for some variables symbolically. These variables can then be split away from the optimization problems such that the iterative numeric methods need to be applied only to the smaller remaining problems.

Assumptions and Proof Obligations. During symbolic calculation in the synthesis kernel, a number of soundness assumptions may accumulate. For example, the expression $x / x$ can be simplified to 1 only if $x \neq 0$ can be shown. Other assumptions stem from the specification or from the applied schemas. Assumptions that cannot be discharged during synthesis are brought to the user's attention. Assumptions which can be checked efficiently during run-time are converted into assertions which are then inserted into the synthesized code (e.g., $x \neq 0$ or $\mathrm{n}_{-}$classes $\ll \mathrm{n}_{\text {_points }}$. This approach ensures soundness and reliability of the generated code.

\section{Examples and Results}

\subsection{Mixture of Gaussians}

In this section, we discuss synthesis and execution of the example described in Section 3. The specification shown in Figure 3 already comprises the entire input to AutoBayes. After parsing the specification, AutoBayes generates the dependency graph (cf. Figure 1) and tries to decompose the original goal

$\underline{\max } \operatorname{pr}(\mathrm{x} \mid\{\mathrm{phi}, \mathrm{mu}$, sigma\}$)$ wrt $\{$ phi, mu, sigma $\}$

into independent parts. In this case, however, the graph is not directly decomposable, and the system tries the match and instantiate one of the statistical algorithm schemas. Here, the EM-schema is applicable and the system identifies c as the single hidden variable, i.e., $W=\{c\}$. For representation of the distribution of the discrete hidden variable $\mathrm{c}$, a matrix $\mathrm{q}$ is generated, where $\mathrm{q}(\mathrm{I}, \mathrm{J})$ is the probability that the $i$-th point falls into the $j$-th class. This array is then initialized using random values. The $E$-step essentially yields a discrete distribution

$c(I) \sim \operatorname{discrete}\left(\operatorname{vec}\left(J:=0 \ldots n_{-} \operatorname{classes}-1, q(I, J)\right)\right.$

For the $M$-step, AutoBAyes is recursively called with the new goal

$\underline{\max } \operatorname{pr}(\{c, x\} \mid\{$ phi, mu, sigma $\})$ wrt $\{$ phi, mu, sigma $\}$

Now, the network decomposition schema described in Section 5.2 applies with $U=$ $\{\mathrm{c}, \mathrm{x}\}, V=\{\mathrm{phi}, \mathrm{mu}$, sigma $\}$, and $X=\{\mathrm{phi}, \mathrm{mu}$, sigma $\}$ which spawns two new subgoals. The first subgoal

$\underline{\max } \operatorname{pr}(\mathrm{c} \mid \mathrm{phi})$ wrt $\{$ phi $\}$ 
can be unrolled over the independent and identically distributed vector c, using an index decomposition schema:

$$
\underline{\max } \prod_{\mathrm{I}:=0}^{\mathrm{n} \text { classes }-1} \operatorname{pr}(\mathrm{c}(\mathrm{I}) \mid \mathrm{phi}) \text { wrt }\{\mathrm{phi}\}
$$

This yields a constrained maximization problem in the vector phi (cf. the constraint with $1=\operatorname{sum}\left(I:=0 \ldots n_{-}\right.$classes $\left.-1, \operatorname{phi}(I)\right)$ in line 10 of the specification) which is solved by an application of the Lagrange multiplier schema. This in turn results in two subproblems for a single instance phi $(j)$ and for the multiplier which are both solved symbolically. The detailed formulas can be found in Figure 7 near Decomposition I.

The second subgoal from the decomposition schema,

$$
\underline{\max } \operatorname{pr}(\mathrm{x} \mid\{\mathrm{c}, \mathrm{mu}, \text { sigma }\}) \text { wrt }\{\mathrm{mu}, \text { sigma }\}
$$

can be unrolled in a similar fashion but since $\mathrm{c}$ and $\mathrm{x}$ are co-indexed, unrolling proceeds over both (also independent and identically distributed) vectors in parallel:

$$
\underline{\max } \prod_{I:=0}^{\mathrm{n} \text {-points-1 }} \operatorname{pr}(\mathrm{x}(\mathrm{I}) \mid\{c(I), \mathrm{mu}, \text { sigma }\}) \text { wrt }\{m u, \text { sigma }\}
$$

The probability $\operatorname{pr}(\mathrm{x}(\mathrm{I}) \mid\{\mathrm{c}(\mathrm{I}), \mathrm{mu}, \mathrm{sigma}\})$ is atomic because parents $(\mathrm{x}(\mathrm{I}))$ $=\{c(I), m u$, sigma $\}$. It can thus be replaced by the appropriately instantiated Gaussian density function:

$$
\underline{\max } \prod_{\mathrm{I}:=0}^{\mathrm{n} \text {-points }-1}(\sqrt{2 \pi} \operatorname{sigma}(\mathrm{c}(\mathrm{I})))^{-1} \exp \left(\frac{(\mathrm{x}(\mathrm{I})-\mathrm{mu}(\mathrm{c}(\mathrm{I})))^{2}}{-2 \operatorname{sigma}(\mathrm{c}(\mathrm{I}))^{2}}\right) \underline{\text { wrt }}\{\mathrm{mu}, \quad \text { sigma }\}
$$

The next step is to "wrap" a log around the formula. This does not change the maximizing values because $\log$ is a strictly monotone function, but it makes the maximization problem easier.

$$
\underline{\max } \sum_{\mathrm{I}:=0}^{\mathrm{n} \text { points }-1}\left(\frac{(\mathrm{x}(\mathrm{I})-\operatorname{mu}(\mathrm{c}(\mathrm{I})))^{2}}{-2 \operatorname{sigma}(\mathrm{c}(\mathrm{I}))^{2}}-\log \sqrt{2 \pi}-\log \operatorname{sigma}(\mathrm{c}(\mathrm{I}))\right) \underline{\text { wrt }}\{\mathrm{mu}, \text { sigma }\}
$$

Now, the hidden variable $\mathrm{c}$ is marginalized using the distribution calculated in the $E$-step. This is accomplished here by summing over the domain of ]tt c, i.e., all possible classes.

$$
\begin{aligned}
& \underline{\max } \sum_{\mathrm{I}:=0}^{\mathrm{n} \text {.points }-1} \sum_{\mathrm{J}:=0}^{\mathrm{n} \_c l a s s e s-1} \mathrm{q}(\mathrm{I}, \mathrm{J})\left(\frac{(\mathrm{x}(\mathrm{I})-\operatorname{mu}(\mathrm{J}))^{2}}{-2 \operatorname{sigma}(\mathrm{J})^{2}}-\log \sqrt{2 \pi}-\log \operatorname{sigma}(\mathrm{J})\right) \\
& \underline{\text { wrt }}\{\mathrm{mu}, \text { sigma }\}
\end{aligned}
$$

This numerical optimization problem for the whole vectors mu and sigma is then simplified by another application of the index decomposition schema into a subproblem for two single instances mu(J) and sigma $(\mathrm{J})$; the fact that both vectors can be unrolled in parallel is again a consequence of the graph structure. In a last step, Gaussian elimination is used to solve this subproblem symbolically, yielding an expression to first calculate $\mathrm{mu}(\mathrm{J})$ and then $\operatorname{sigma}(\mathrm{J})$.

$$
\begin{aligned}
& \mathrm{mu}(\mathrm{J})=\sum_{\mathrm{I}:=0}^{\mathrm{n} \_ \text {points }-1}(1 / \mathrm{q}(\mathrm{I}, \mathrm{J})) \sum_{\mathrm{K}:=0}^{\mathrm{n} \text { _points }-1} \mathrm{x}(\mathrm{K}) \mathrm{q}(\mathrm{K}, \mathrm{J}) \\
& \operatorname{sigma}(J)=\sum_{I:=0}^{n_{\text {ppoints }}-1}(1 / \sqrt{\mathrm{q}(\mathrm{I}, \mathrm{J})}) \sum_{\mathrm{K}:=0}^{\mathrm{n}_{\mathrm{N}} \text { points }-1} \sqrt{\mathrm{q}(\mathrm{K}, \mathrm{J})}(\mathrm{x}(\mathrm{K})-\mathrm{mu}(\mathrm{K}))^{2}
\end{aligned}
$$


(a)

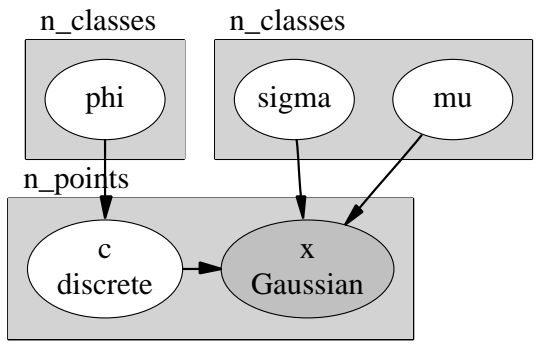

(b)

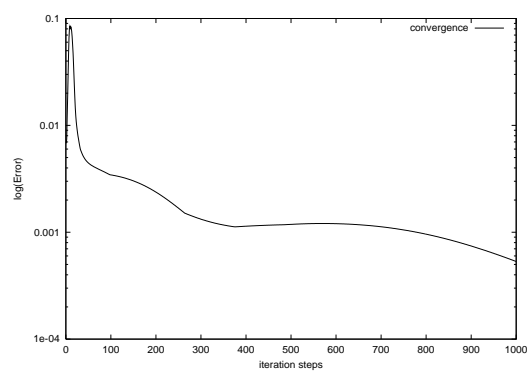

Fig. 5. (a) Bayesian network for the mixture of Gaussians example, automatically generated by AutoBAyEs from the textual specification; (b) convergence behavior: differences between old and new parameters (log-scale) over iteration step. Only the first 1000 iteration cycles are shown.

For the entire example, AutoBayes synthesizes a C++ file consisting of 389 lines, including comments and separation lines. A portion of this code is shown in Figure 7. The code is then compiled into a dynamically linkable function for Octave. Thus, when the function mog (cf. line 1 of the specification) is invoked within the Octave environment, the compiled $\mathrm{C}++$ code is invoked automatically. As shown in a sample run in Figure 6, AutoBAYES also synthesizes code to show the required input- and output parameters ("usage"). The entire synthesis process of AutoBayes, including compilation of the generated $\mathrm{C}++$ code takes about 25 secs. on a $400 \mathrm{Mhz}$ Sun Ultra 60 . For further details, see problem $M_{1}$ in Table 1.

We have tested the synthesized code with artificial test data which has been generated by the test data generator synthesized by AutoBAYes from the same model. The data set consists of 2400 points divided into 3 classes (cf. Figure 2). From these inputs, the algorithm searches for the values of mu, sigma, and phi for each class. The convergence, i.e., the normalized change of the parameters to be optimized during each iteration cycle, is shown in Figure 5. This algorithm does not necessarily converge monotonically. It can reach some local minimum, from which it has to move away by increasing the error again. After some ups and downs the global minimum (i.e., an optimal estimate for the parameters) is reached and the loop ends. This behavior is typical for many iterative parameter estimation processes. In this case, the final result required 1163 iteration steps, taking approximately 48 secs. $^{2}$ AutoBayes can automatically instrument the generated code to produce these run-time figures for debugging and testing purposes if this is requested via a command line option.

\subsection{Other Examples}

We have also applied the AutoBAYEs system to a number of different textbook and benchmark examples. The results of these experiments are shown in Table 1.

2 These figures can change from run to run, since the algorithm starts with a random initial class assignment for each data point. 


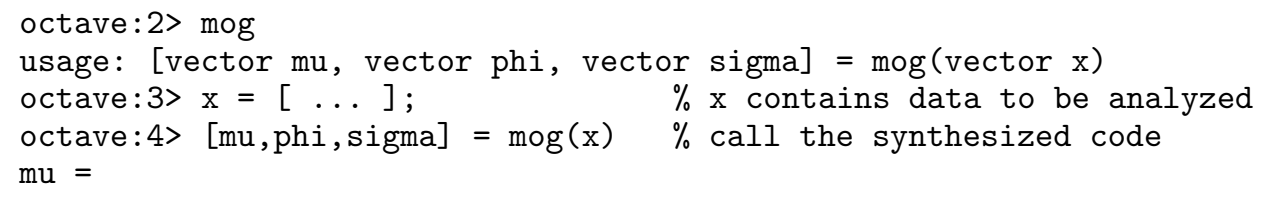

Fig. 6. Octave sample session using code (function "mog") generated by AutoBAYES

For each problem, a short description of the task or the used priors is given. $c f s$ indicates whether a closed-form solution exists and, if so, whether it has been found by AutoBayes. The next two columns give the size of the specification and the respective number of lines of generated Octave/C++ code, including the automatically generated comments. Finally, the synthesis time $T_{\text {synth }}$ (i.e., AutoBayes's run-time) as well as the compilation time $T_{\text {compile }}$ for the GNU $\mathrm{g}^{++}$compiler (optimization level -02) are given. All times are in seconds and have been obtained on a Sun Ultra 60 (400 Mhz) using the Unix time command.

The examples $G_{1}$ to $G_{4}$ describe different estimation problems for Gaussian distributions. Given a sample of $n$ data points and various prior information (e.g., the variance of the distribution and an estimate of the mean value), the task is to estimate the remaining parameters of the distribution. For most of these textbook examples closed-form solutions exist (Gelman et al., 1995) and are found by AutoBayes, which demonstrates the capabilities of its symbolic system. The examples $G_{3}$ and $G_{4}$ also demonstrate how small changes in the specification can lead to dramatically different programs. $G_{3}$ uses the so-called conjugate prior for $\mu$ and can still be solved in closed form. In $G_{4}$, however, the slightly more general semiconjugate prior is used (i.e., the variance of the expected mean is generalized from the form $\left(\sigma^{2} / \kappa_{0}\right)^{0.5}$ to a simple variable $\left.\tau_{0}\right)$ which renders the problem unsolvable in closed form and, hence, requires the application of an iterative approximation method, in this case, a Nelder-Mead simplex algorithm.

We have also been able to synthesize code for a large number of mixture problems. $M_{1}$ is the example problem used throughout this paper. Variations of the Mixture of Gaussians problem for uncorrelated two-dimensional observations $\left(M_{2}\right)$ and for hidden variables composed from multiple independent dimensions $\left(M_{3}\right)$ as well as most of the problems given in the textbook (Everitt \& Hand, 1981) on mixture problems have been tried out. All mixture problems are solved by different instantiations of the EM-schema; however, the different distributions give rise to different maximization problems in the M-step. An efficient implementation requires the symbolic solution of the emerging maximization problem. AUTOBAYES' symbolic system is already powerful enough to provide such solutions for the distributions from the exponential family, including the binomial, exponential $\left(M_{4}\right)$, Gaussian, and Poisson distributions. 
AutoBayes: Generating Data Analysis Programs from Statistical Models

\begin{tabular}{|c|c|c|c|c|c|}
\hline$\#$ & $\begin{array}{l}\text { Description } \\
\text { (priors) }\end{array}$ & $\mathrm{cfs}$ & $\begin{array}{l}\text { lines } \\
\text { spec }\end{array}$ & $\begin{array}{l}\text { code } \\
\mathrm{C}++\end{array}$ & $\begin{array}{l}T_{\text {synth }}[s]+ \\
T_{\text {compile }}[\mathrm{s}]\end{array}$ \\
\hline$G_{1}$ & $\mu \sim \operatorname{gauss}\left(\mu_{0}, \tau_{0}^{0.5}\right), \sigma^{2}$ & $\mathrm{Y} / \mathrm{Y}$ & 12 & 99 & $1.5+7.1$ \\
\hline$G_{2}$ & $\mu, \sigma^{2} \sim \Gamma^{-1}\left(\delta_{0} / 2+1, \sigma_{0}^{0.5} \delta_{0} / 2\right)$ & $\mathrm{Y} / \mathrm{Y}$ & 13 & 99 & $2.0+8.8$ \\
\hline$G_{3}$ & $\begin{array}{l}\mu \sim \operatorname{gauss}\left(\mu_{0},\left(\sigma^{2} / \kappa_{0}\right)^{0.5}\right) \\
\sigma^{2} \sim \Gamma^{-1}\left(\delta_{0} / 2+1, \sigma_{0}^{0.5} \delta_{0} / 2\right)\end{array}$ & $\mathrm{Y} / \mathrm{Y}$ & 17 & 126 & $8.9+7.7$ \\
\hline$G_{4}$ & $\begin{array}{l}\mu \sim \operatorname{gauss}\left(\mu_{0}, \tau_{0}\right) \\
\sigma^{2} \sim \Gamma^{-1}\left(\delta_{0} / 2+1, \sigma_{0}^{0.5} \delta_{0} / 2\right)\end{array}$ & $\mathrm{N} / \mathrm{N}$ & 17 & 478 & $14.6+20.0$ \\
\hline$M_{1}$ & 1D Gaussian mixture & $\mathrm{N} / \mathrm{N}$ & 16 & 389 & $11.7+12.4$ \\
\hline$M_{2}$ & $\begin{array}{l}\text { 2D Gaussian mixture } \\
(x, y \text { uncorrelated })\end{array}$ & $\mathrm{N} / \mathrm{N}$ & 22 & 536 & $19.6+19.7$ \\
\hline$M_{3}$ & $\begin{array}{l}\text { 1D Gaussian mixture } \\
\text { (multi-dimensional classes) }\end{array}$ & $\mathrm{N} / \mathrm{N}$ & 24 & 519 & $18.1+16.7$ \\
\hline$M_{4}$ & $\begin{array}{l}\text { exponential mixture } \\
\text { (simple failure analysis) }\end{array}$ & $\mathrm{N} / \mathrm{N}$ & 15 & 321 & $6.4+10.0$ \\
\hline$M_{5}$ & disjoint mixture & $\mathrm{N} / \mathrm{N}$ & 21 & 425 & $19.5+11.9$ \\
\hline$M_{6}$ & $1 \mathrm{D}$ mixture $\mathrm{w} /$ priors on $\sigma$ & $\mathrm{N} / \mathrm{N}$ & 20 & 401 & $15.4+15.0$ \\
\hline$M_{7}$ & $1 \mathrm{D}$ mixture $\mathrm{w} /$ priors on $\mu$ & $\mathrm{N} / \mathrm{N}$ & 24 & 424 & $18.2+16.5$ \\
\hline $\mathrm{SD}$ & step detection & $\mathrm{N} / \mathrm{N}$ & 14 & 1206 & $78.0+49.4$ \\
\hline $\mathrm{AB}$ & Abalone classifier & $\mathrm{N} / \mathrm{N}$ & 58 & 1310 & $63.5+139.1$ \\
\hline
\end{tabular}

Table 1. List of examples

AutoBayes can easily be extended to handle more complicated mixture models. For example, we have added a higher-order mixture-operator to handle nonparametric mixtures, i.e., models in which the different classes are generated by different probability distribution functions and not only by different parameter values of the same distribution. The mixture-operator simply takes a finite list of the different distributions and mixes them according to the value of the hidden variable, e.g.,

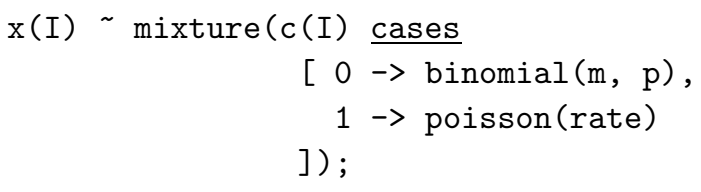

describes the mixture between a binomial and a Poisson process used in example $M_{5}$. Due to the schema-based approach, this extension was completely straightforward and required only two additional Prolog-clauses, one to declare mixture as the name of a distribution and one to define its distribution function as a casesconstruct over the distribution functions of its arguments. In particular, no further functionality specific to the mixture-operator needed to be implemented. Finally, $M_{6}$ and $M_{7}$ are one-dimensional mixture examples with prior information (conjugate prior) on $\sigma$ and $\mu$, respectively. These examples demonstrate AuTOBAYES's capability to synthesize code for classical (i.e., without priors) maximum-likelihood problems as well as for maximum aposteriori (i.e., Bayesian inference) problems.

The step detection problem $S D$ is to estimate the time at which the mean of a Gaussian process changes. Such a change can indicate a failure in the underlying physical process. The change can easily be specified in AutoBayes: 
$\mathrm{x}(\mathrm{I}) \sim \operatorname{gauss}(\underline{\mathrm{if}}(\mathrm{I}<\mathrm{step}, \mathrm{mu} 1, \mathrm{mu} 2)$, sigma $)$;

There are several algorithms for step detection. One of the more common approaches is the Hinckley-test which first finds the maximizing values for mu1, mu2 and sigma in terms of the still unknown position step, substitutes these values back into the original problem, and then finds the maximizing value for step. AutoBAYES "re-invents" this algorithm by composition of three different schemas, a split/backsubstitute schema for separating the problem, range-iteration for solving the discrete subproblem, and the symbolic solver for handling the continuous part.

The Abalone classification problem $A B$ is a standard machine learning benchmark from the UCI Machine Learning Repository (Blake \& Merz, 1998). Here, the age of an Abalone mussel has to be predicted from a number of physical measurements, e.g., its size or weight. Prediction is used because an exact age determination requires an elaborate procedure - cutting the shell, staining it, and counting the number of rings through a microscope. In its original form, the age prediction is a difficult problem because the data set contains only very few entries for very young or very old abalones. It is thus often simplified by partitioning the ages into three roughly equally likely categories "young," "adult," and "old." For this simplified version, AutoBayes generates an unsupervised classifier (i.e., no training phase is required) which is again based on the EM-schema. It achieves a $54.7 \%$ accuracy which is only slightly worse than the results of some of the supervised classifiers.

In general, these results are very encouraging as they indicate that AUTOBAYES can already be applied to realistic examples. Except for the last two examples, synthesis times are generally in the sub-minute range; they also compare well with the compile times for the synthesized code. Most of the synthesis time is generally spent in the symbolic subsystem which we believe can still be optimized substantially. The only exception here is the step detection example $S D$ where almost $90 \%$ of the synthesis time is spent in the backend. This is a result of the large number of deeply nested summations which are converted into loops and thus require a substantial re-arrangement of the code. In the cases where no closed-form solution exists, the scale-up factor (i.e., the ratio between specification size and code size) is generally around 1:20 which supports our claim that models are much more concise than programs.

We are currently testing AutoBAyes in two larger case studies concerning data analysis tasks for finding extra-solar planets, either by measuring dips in the luminosity of stars (Koch et al., 2000), or by measuring Doppler effects (Marcy \& Butler, 1997), respectively. Both projects required substantial effort to manually set up data analysis programs. Our goal for the near future is to demonstrate AuTOBAYES's capability to handle major subproblems (e.g., the CCD-sensor registration problem) arising in these projects.

\section{Related Work}

AutoBayes combines two different fields, statistics and program synthesis. Consequently, related work can be found in both fields. In statistics, there is a long 
tradition of composing programs from library components but there are only a few, recent attempts to achieve a similar degree of automation as AuTOBAYEs does. The Bayes Net Toolbox (Murphy, 2000) is a Matlab-extension which allows users to program in models; it provides several Bayesian inference algorithms which are attached to the nodes of the network. However, the Toolbox is a purely interpretive system and does not generate programs. The widely used BuGs-system (Thomas et al., 1992) also allows users to program in models but it uses yet another, entirely different execution model: instead of executing library code or generating customized programs, it interprets the statistical model using Gibbs sampling, a universal-but less efficient_Bayesian inference technique. Gibbs sampling could be integrated into AutoBAyes as an algorithm schema. Mjolsness and Turmon (Mjolsness \& Turmon, 2000) recently introduced the concept of stochastic parameterized grammars. Such grammars allow a concise model specification in a way very similar to AutoBAyes's specification language. However, they are currently only a notational device without any underlying program execution or synthesis model.

Deductive synthesis is still an active research area, despite its long heritage going back to (Green, 1969) and (Waldinger, 1969). Some systems, however, have already been applied to real-world problems. The AMPHION system (Stickel et al., 1994) has been used to assemble programs for celestial mechanics from a library of FORTRAN components, for example the simulation of a Saturn fly-by. AmPHION is more component-oriented than AutoBAYES, i.e., the generated programs are linear sequences of subroutine calls into the library. It uses a full-fledged theorem prover for first-order logic and extracts the program from the proof. Ellman and Murata (Ellman \& Murata, 1998) describe a system for the deductive synthesis of numerical simulation programs. This system also starts from a high-level specification of a mathematical model - in this case a system of differential equations - but is again more component-oriented than AUTOBAYES and does not use symbolicalgebraic reasoning. Planware (Blaine et al., 1998) (which grew out of the KIDS system (Smith, 1990)) synthesizes schedulers for military logistics problems. It is built on the concept of an algorithm theory which can be considered as an explicit hierarchy of schemas, but the underlying basic synthesis process is a different one.

Biggerstaff (Biggerstaff, 1999) presents a short classification of generator techniques (albeit cast in terms of their reuse effects). AuTOBAYEs falls most closely into the category of inference-based generators but also exhibits some aspects of pattern-directed and reorganizing generators, e.g., the typical staging of the schemas into multiple levels.

\section{Conclusions and Future Work}

We have presented AutoBAYEs, a system for the automatic synthesis of data analysis programs from specifications in the form of statistical models. AuTOBAYES follows a schema-guided deductive synthesis approach. After constructing the initial Bayesian network from the given specification (i.e., the statistical model), a variety of different schemas are tried exhaustively. These schemas are guarded by applicability constraints and contain code-blocks which are instantiated. By way of 
an intermediate language, AutoBAyEs generates executable, optimized code for a target system. The current version produces $\mathrm{C} / \mathrm{C}++$-code which can be linked dynamically into the Octave and Matlab environments. We have tested AuTOBAYES on a variety of text-book and benchmark examples. In most cases, run-time for synthesizing code was well below one minute; compiling the synthesized codes takes roughly the same amount of time. The code is well documented and robust.

Although we have been able to generate code for various non-trivial textbook examples, AutoBAyes's capabilities to generate code for a variety of statistical models must be extended, before it can be employed by the working data analyst. We will add further algorithm schemas for statistical algorithms (e.g., variants of the $E M$-algorithm) and for general numerical optimization to the system. Future versions of AUTOBAYES will also be extended in such a way that statistical models over time series can be handled. Here, we are planning to incorporate specific algorithm schemas for handling a restricted but common class of time-series problems as well as standard optimization methods like finite differencing.

AutoBAYEs offers several unique features which result from using program synthesis instead of compilation and which make it more powerful and more versatile for the application domain than other tools and statistical libraries. AuTOBAYES can generate efficient procedural code from a high-level, declarative specification without any notion of data-flow or control-flow. Thus, it covers a relatively large semantic gap between specification and code and provides substantial leverage. Due to the concise semantics of the specifications and the domain theory, the synthesized code is provably correct and always consistent with the specification. Synthesis times are very short. Changes and modifications of the statistical model can thus be applied without time-consuming re-implementation of the data analysis program. Such fast turn-around times are particularly valuable for iterative software engineering processes as well as for science applications where the underlying models are not yet well understood. By combining schema-guided synthesis with symbolic calculation, AutoBAyEs can find closed-form solutions for many problems. Thus, the generated code for these kinds of problems is extremely efficient and accurate, because it does not rely on numeric approximations.

AutoBAyEs can generate different programs for the same specification. Although the overall functionality of each of the synthesized programs is the same (i.e., as given in the specification), they can differ substantially with respect to speed, numerical stability and memory consumption. This feature is based on exhaustive search and application of algorithm schemas and is naturally supported by Prolog's backtracking mechanism. It allows the user to effectively explore the design space. In combination with AutoBAyEs's test data generator the user is thus able to select a synthesized program which best fits the given application profile. For future versions of AUTOBAYES we aim to incorporate user-defined design constraints to control the search process. The explanation technique offers major benefits, especially for safety-critical areas. Code is not only documented for human understanding, but assumptions made in the specification and during synthesis are checked by assertions during run-time. This makes the generated code more robust against erroneous inputs or faulty data. 
AutoBayes is still an experimental system and must still be extended in various ways. In particular, the domain coverage of AUTOBAYES must be increased to handle more complex models. Nevertheless, we are confident that the paradigm of schema-guided synthesis is an appropriate approach to program generation in this domain and will lead to a powerful yet easy-to-use tool.

Acknowledgements: Wray Buntine and Tom Pressburger contributed much to the initial development of AutoBayes. Grigore Rosu implemented the test data generator and the graph visualization. We also want to thank the anonymous reviewers for their helpful comments.

\section{References}

AutoCode. (1999). MatrixX: AutoCode Product Overview. ISI.

Berkowitz, Joseph. (1979). Photoabsorption, photoionization, and photoelectron spectroscopy. Academic Press.

Biggerstaff, Ted J. (1999). Reuse technologies and their niches. Pages 613-614 of: Garlan, David, \& Kramer, Jeff (eds), Proc. 21th intl. conf. software engineering. Los Angeles, CA: ACM Press. Extended abstract.

Bishop, Ch. M. (1995). Neural networks for pattern recognition. Oxford: Clarendon-Press.

Blaine, Lee, Gilham, Li-Mei, Liu, Junbo, Smith, Douglas R., \& Westfold, Stephen. (1998). Planware - domain-specific synthesis of high-performance schedulers. Pages 270-280 of: Redmiles, David F., \& Nuseibeh, Bashar (eds), Proc. 13th intl. conf. automated software engineering. Honolulu, Hawaii: IEEE Comp. Soc. Press.

Blake, C.L., \& Merz, C.J. (1998). UCI repository of machine learning databases.

Buntine, Wray L. (1994). Operations for learning with graphical models. J. ai research, 2, 159-225.

Buntine, Wray L., Fischer, Bernd, \& Pressburger, Thomas. (1999). Towards automated synthesis of data mining programs. Pages 372-376 of: Chaudhuri, Surajit, \& Madigan, David (eds), Proc. 5th intl. conf. knowledge discovery and data mining. San Diego, CA: ACM Press.

Centre for Atmospheric Science. (1999). The ozone hole tour.

ControlShell. (1999). ControlShell. RTI Real-Time Innovations.

Dempster, A. P., Laird, N. M., \& Rubin, Donald B. (1977). Maximum likelihood from incomplete data via the EM algorithm (with discussion). J. of the royal statistical society series b, 39, 1-38.

Ellman, Thomas, \& Murata, Takahiro. (1998). Deductive synthesis of numerical simulation programs from networks of algebraic and ordinary differential equations. Automated software engineering, 5(3), 291-319.

Everitt, B. S., \& Hand, D. J. (1981). Finite mixture distributions. Monographs on Applied Probability and Statists. London: Chapman \& Hall.

Fischer, Bernd, Schumann, Johann, \& Pressburger, Thomas. (2000). Generating data analysis programs from statistical models (position paper). Pages 212-229 of: Taha, Walid (ed), Proc. intl. workshop semantics applications, and implementation of program generation. Lect. Notes Comp. Sci., vol. 1924. Montreal, Canada: Springer.

Frey, Brendan J. (1998). Graphical models for machine learning and digital communication. Cambridge, MA: MIT Press.

Gelman, Andrew, Carlin, John B., Stern, Hal S., \& Rubin, Donald B. (1995). Bayesian data analysis. Texts in Statistical Science. Chapman \& Hall. 
Gill, Philip, Murray, Walter, \& Wright, Margaret. (1981). Practical optimization. Academic Press.

Green, Cordell. (1969). Application of theorem proving to problem solving. In: (Walker \& Norton, 1969).

Jordan, Micheal I. (ed). (1999). Learning in graphical models. Cambridge, MA: MIT Press.

Koch, D. G., Borucki, W., Dunham, E., Jenkins, J., Webster, L., \& Witteborn, F. (2000). CCD photometry tests for a mission to detect earth-size planets in the extended solar neighborhood. Proceedings spie conference on uv, optical, and ir space telescopes and instruments.

Koutsofios, Eleftherios, \& North, Stephen. 1996 (Nov.). Drawing graphs with dot. Tech. rept. AT\&T Bell Laboratories, Murray Hill, NJ, USA.

Marcy, G. W., \& Butler, R. P. (1997). Extrasolar planets detected by the doppler technique. Proceedings of workshop on brown dwarfs and extrasolar planets.

McLachlan, Geoffrey, \& Krishnan, Thriyambakam. (1997). The EM algorithm and extensions. Wiley Series in Probability and Statistics. New York: John Wiley \& Sons.

McLachlan, Geoffrey, \& Peel, David. (2000). Finite mixture models. Wiley Series in Probability and Statistics. New York: John Wiley \& Sons.

Mitchell, Tom. (1997). Machine learning. McGraw Hill.

Mjolsness, Eric, \& Turmon, Micheal. 2000 (Dec.). Stochastic parameterized grammars for bayesian model composition. Buntine, Wray, Fischer, Bernd, \& Schumann, Johann (eds), Nips*2000 workshop on software support for bayesian analysis systems.

Moler, C. B., Little, J. N., \& Bangert, S. (1987). PC-Matlab users guide. Cochituate Place, 24 Prime Park Way, Natick, MA, USA.

Murphy, Kevin. (2000). Bayes net toolbox 2.0 for matlab 5. http://www.cs. berkeley.edu/ murphyk/Bayes/bnt.html.

Murphy, Malcolm. (1997). Octave: A free, high-level language for mathematics. Linux journal, 39 (July).

Pearl, Judea. (1988). Probabilistic reasoning in intelligent systems: Networks of plausible inference. San Mateo, CA, USA: Morgan Kaufmann Publishers.

Press, William H., Flannery, Brian P., Teukolsky, Saul A., \& Vetterling, William T. (1992). Numerical recipes in C. 2nd. edn. Cambridge, UK: Cambridge Univ. Press.

Smith, Douglas R. (1990). KIDS: A semi-automatic program development system. Ieee trans. software engineering, 16(9), 1024-1043.

Stickel, Mark, Waldinger, Richard, Lowry, Michael, Pressburger, Thomas, \& Underwood, Ian. (1994). Deductive composition of astronomical software from subroutine libraries. Pages 341-355 of: Bundy, Alan (ed), Proc. 12th intl. conf. automated deduction. Lect. Notes Artifical Intelligence, vol. 814. Nancy: Springer.

Thomas, A., Spiegelhalter, D. J., \& Gilks, W. R. (1992). BUGS: A program to perform Bayesian inference using Gibbs sampling. Pages 837-842 of: Bernardo, J. M., Berger, J. O., Dawid, A. P., \& Smith, A. F. M. (eds), Bayesian statistics 4. Oxford Univ. Press.

Waldinger, Richard J. (1969). PROW: a step towards automatic program writing. In: (Walker \& Norton, 1969).

Walker, Donald E., \& Norton, Lewis M. (eds). (1969). Proc. 1st intl. joint conf. artificial intelligence. Washington, DC: William Kaufmann.

Wielemaker, Jan. (1998). SWI-prolog 3.1 reference manual, updated for version 3.1.0 july, 1998. Amsterdam. 


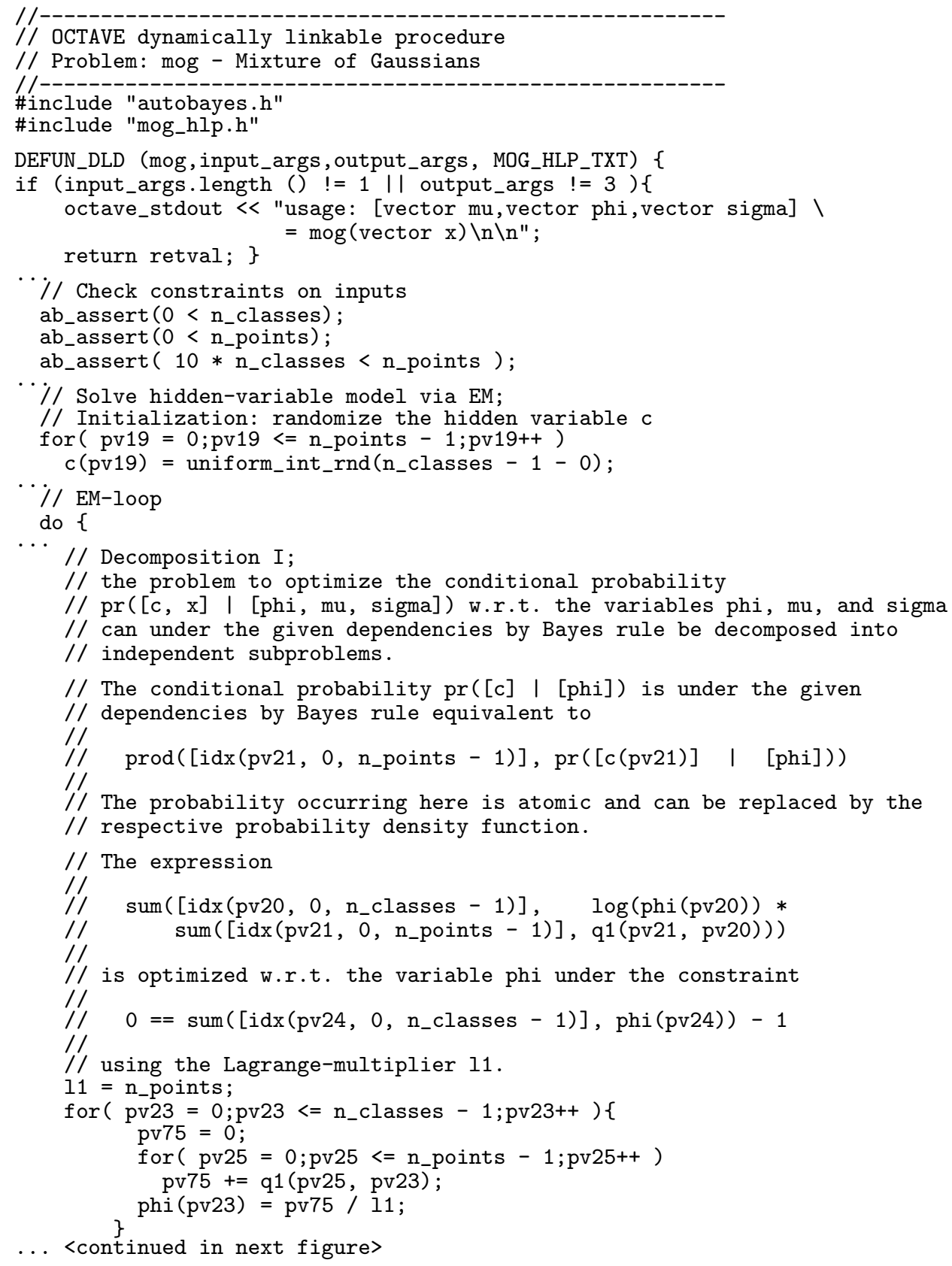

Fig. 7. C++-code for the Mixture of Gaussians example (excerpts). 


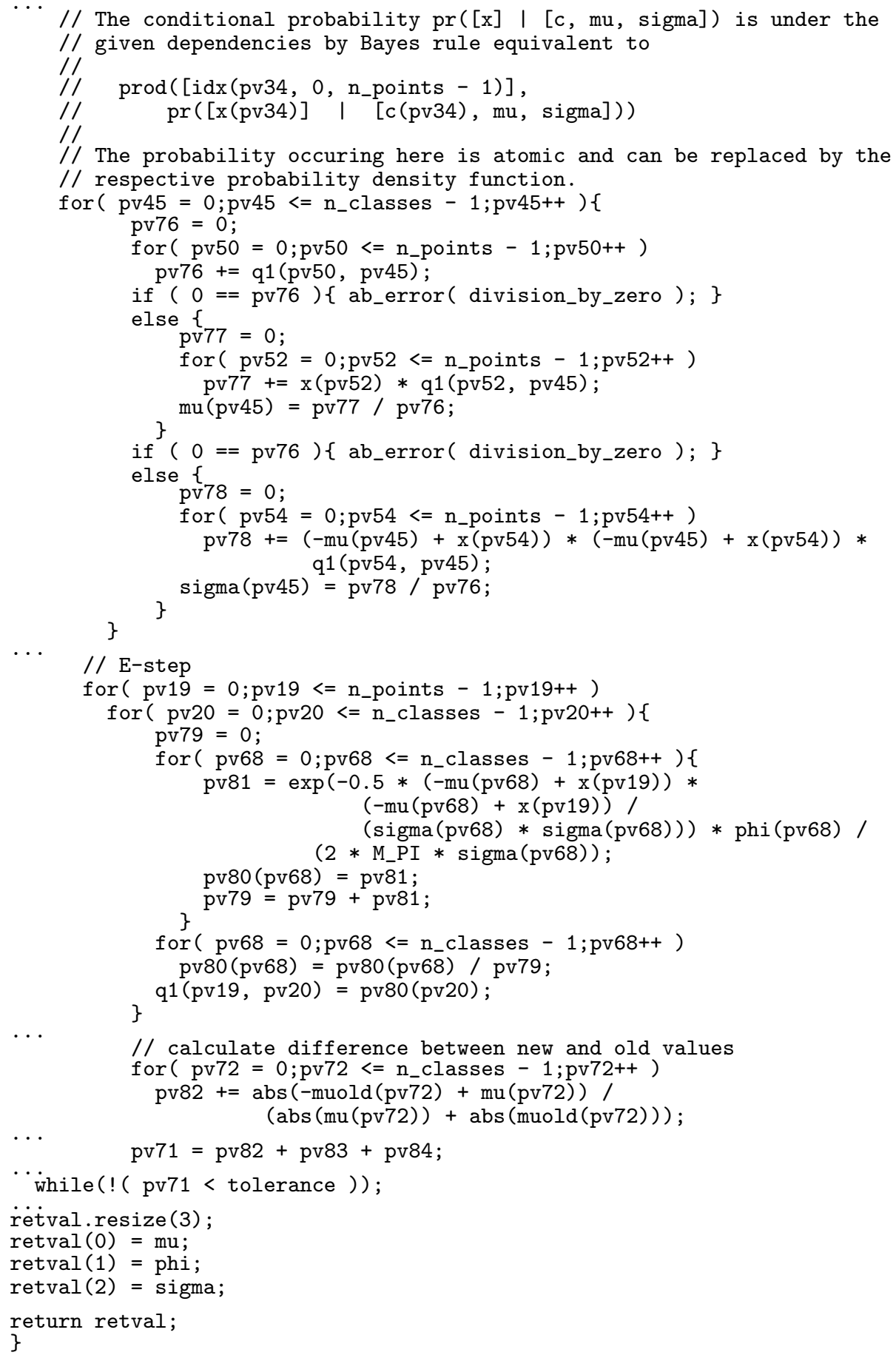

Fig. 8. C++-code for the Mixture of Gaussians example (continued). 